\title{
Measuring the Quality of Urban Environments: A Need-Based Micro-Simulation Approach
}

\author{
Theo A. Arentze • Peter J. H. J. van der Waerden • \\ Jochem W. Bergen • Harry J. P. Timmermans
}

Received: 13 September 2007 / Accepted: 15 April 2009 /

Published online: 19 May 2009

(C) The Author(s) 2009. This article is published with open access at Springerlink.com

\begin{abstract}
The ability of an urban environment to support the daily activities individuals need or wish to conduct in time and space has received attention particularly in constraints-based approaches. These approaches do, however, not consider needs underlying an individual's activities. In this paper, we develop a new measurement approach that is based on a needs-based theory that we developed in earlier work. In this approach, the quality of an environment is measured based on a representative set of daily activity patterns in a studied population. Activity patterns are spatially represented as route systems in a GIS environment and attributes of stops and routes are measured using standard GIS tools. Using the needs-based model, the extent to which each activity pattern satisfies a set of needs is measured as a function of mode and duration-dependent exposure to these attributes of the environment. The aggregated need-satisfaction across activity patterns is taken as a measure of quality. We illustrate the method in a case study where we use TransCAD for spatial analysis and an existing activity diary dataset from the Eindhoven region and where we focus on green-recreation needs. The case study shows the sensitivity of the measure for spatial scenarios regarding availability and spatial distribution of green area in the city.
\end{abstract}

Keywords Accessibility - Urban planning - Spatial performance indicators · Activity-based modeling $\cdot$ Needs theory

\section{Introduction}

The opportunities an urban environment offers to its residents constitute a major concern in urban planning. An important aspect of spatial opportunities is the

T. A. Arentze $(\bowtie) \cdot$ P. J. H. J. van der Waerden • J. W. Bergen • H. J. P. Timmermans Urban Planning Group, Eindhoven University of Technology, PO Box 513, 5600 MB Eindhoven, The Netherlands e-mail: t.a.arentze@bwk.tue.nl e-mail: eirass@bwk.tue.nl 
accessibility of locations, which is generally defined as the relative ease by which the locations of activities, such as for example working, shopping, recreation and other service-based activities, can be reached from a given location. Originally, measures of accessibility have been based on average distance or travel time to conduct a particular activity (e.g. Ingram 1971; Breheney 1978). More sophisticated measures have used some distance decay function or welfare measure, originally based on the spatial interaction model, such as log-accessibility (Leonardi 1978) and consumer surplus (Coelho and Wilson 1976), and later on discrete choice models (Ben-Akiva and Lerman 1978). These approaches are reviewed in Dalvi (1978) and Koenig (1980). Alternatively, measures based on the notion of accumulated opportunities (Ingram 1971; Breheney 1978; Wachs and Kumagai 1973) sometimes taking into account trip-chaining behavior (Arentze et al. 1994a, b) have been suggested.

These single-activity-based accessibility measures are informative, but do not take into account the influence of temporal factors on the feasibility of daily activity patterns. Individuals have limited time budgets and activities are often constrained in terms of start time and duration and moreover require travel time. To take into account such temporal factors, the concept of accessibility has been extended in a more recently introduced activity-based approach which has its roots in Hagerstrand's time geography (e.g., Miller 1999; Kwan 1998, 1999; Dijst et al. 2002; Kim and Kwan 2003; Weber 2003). In this so-called constraints-based approach, the space-time opportunities an environment offers is measured as the extent to which it allows individuals to implement complete activity patterns on a daily and individual basis (Lenntorp 1978; Jones at al. 1983).

Although the constraints-based approach provides a comprehensive measure of accessibility, it does not address quality in terms of the pleasure or utility an individual derives from an urban environment while implementing an activity pattern. In this paper, we propose a new method to measure the quality of an urban environment that complements the (activity-based) concept of space-time opportunities. As in constraints-based approaches, the new method takes full activity schedules of individuals and associated temporal constraints into account. Complementary to the concept of accessibility, however, it highlights the notion that urban environments to varying extents satisfy particular needs of people while they implement activities in time and space. Therefore, in addition to the question 'to what extent does a space-time environment support the activities people conduct?', the method we propose addresses the question 'to what extent does a space-time environment satisfies various needs individuals may have while they interact with their environment?'.

To operationalize this concept, the method we propose is based on the need-based model of activity generation that we developed in earlier work (Arentze and Timmermans 2006, 2009). This model describes how basic needs of an individual/ household change over time as a function of the activities of the individual/ household and attributes of the environment. The utility function of activity patterns takes into account (negative or positive) substitution relationships between activities and saturation effects.

In this paper, we first derive the proposed measurement method from the needbased activity model. Next, we show how activity patterns can be represented as 
route systems in the GIS-software TransCAD and how need-based utility functions can be implemented in this GIS environment. In a case study, we apply the method to the Eindhoven region based on a large sample of activity patterns collected through an activity-diary survey. We apply the method to analyze the quality of the urban environment for the existing situation and a spatial policy scenario to illustrate the method and demonstrate its sensitivity as a measurement instrument.

\section{Basic Concepts and Measurement Approach}

In this section, we discuss the proposed measuring method in terms of basic concepts and approach. The concepts are based on a needs-based theory and operational model of activity generation that we developed in earlier work referred to above. The model consists of a system of equations that defines the mutual relationships between activities, needs and utilities and an activity scheduling heuristic. We first discuss this framework focusing on the elements that are relevant for the proposed measurement approach. Next, we discuss how the model can be used for the measurement purpose.

\section{The Need-Based Theory of Activity Generation}

The theory assumes that the activities people conduct in space and time are driven by a limited set of universal needs. The dynamics of needs and limited time budgets of individuals explain the repertoire and rhythmic character of activities through time. We do not make assumptions regarding which needs exist, but rather view this as a matter of empirical research. However, needs is a very general concept that has been defined in many different ways dependent on theoretical background. A helpful notion to clarify the way we conceptualize it is the target need of an activity. Each activity an individual conducts on a regular basis tends to be focused on a particular need. The target need is the reason of existence of the activity. Each activity in an individual's repertoire is, as it were, designed to satisfy a need on a particular dimension and together they should cover needs exhaustively. For example, the target of shopping is a need for restocking daily or non-daily goods, the target of visiting a friend is a need for social interaction, the target of green recreation is a need for being in a green environment, etc. At the same time, our theory acknowledges that there may not be a one-to-one correspondence between needs and activities. An activity may also produce effects - positive or negative - on other than its target need. For example, a shopping activity may to some extent satisfy a need for entertainment (e.g., watching shop windows), for physical exercise (e.g., walking around), for social interaction (e.g., meeting other people), for green recreation (e.g., being in the open air), etc. Such side effects may also be negative. For example, a work activity may increase a need for resting (if it is tiresome) or increase a need for entertainment (if it is boring).

Needs are not static but change (usually, grow) over time independent of specific activities conducted, given the level of abstraction on which we consider activities. This growth may represent physiological processes in case of more elementary needs (e.g., a need for food grows due to combustion of nutrients in the body), but it may 
also represent factors of activities not accounted for. For example, although a need for re-stocking goods will be induced by consumption activities of individuals in a household, a smooth growth function is assumed if those consumption activities are not explicitly represented in the system.

Each activity conducted by an individual has an impact on one or more needs; it will reduce the target need and, as side effects, may decrease or increase other needs at the same time. The direction of the impacts is determined by the nature of the activity, whereas the size of the impacts depends on the activities duration and attributes of the location where the activity is conducted. The longer the duration the longer the time the activity inserts its impact and, hence, the larger the overall impact will be. Attributes of the location, on the other hand, will influence the extent to which the activity is capable of satisfying the need, given its duration ${ }^{1}$. In the model, traveling is considered an activity just as an activity at a location. In terms of impacts on needs, the model treats travel time, travel mode and route of a trip as equivalents to activity duration, activity type and activity location for stationary activities. Within-household interactions between individuals are also relevant if a subset of needs is shared by persons in a same household. Activities conducted by one person that affect household-level needs will have an impact on needs of other persons as well. For example, a housekeeping activity may satisfy a need for a clean and tidy house that all persons share. Although the needs-based framework incorporates this aspect, we will disregard it in the following for reasons of simplicity.

For the current measurement purpose, we specify the model for a following representation of the problem. We consider a specific individual and a specific day and assume an activity pattern of the individual for that day as given. An activity pattern describes a particular sequence of activity and travel episodes. Each episode (activity or trip) $j$ is described by a duration (travel time or activity time), $T_{j}$, a set of attributes, $X_{j}$, of the location (activity) or route (travel) involved and a mode of behavior, $A_{j}$, which indicates an activity type (activity) or transport mode (travel). Following a basic assumption of the need-based theory, the utility an individual derives from his or her activity pattern, is defined as:

$$
U=-\sum_{i \in I, j \in J} \Delta B_{i j}
$$

where $U$ is the utility derived from the activity pattern, $I$ is a universal set of needs, $J$ is the set of activities (including travel) conducted and $\Delta B_{i j}$ is the change of need $i$ caused by activity $j$. In words, the perceived utility of an activity pattern equals the sum of need changes across activities and needs. Utility is positive if more needs are satisfied than induced and zero or negative otherwise. Activities and travel divide the day of an individual into different episodes. We use the symbol $t$ as an index of time, where $t=0$ is the start time of the day and $t=\Sigma_{j} T_{j}$ is the end time of the day (i.e., there are no time gaps or time overlaps between episodes). The size of need $i$ of the individual considered at the start of the day, $B_{i}{ }^{0}$, is assumed as given.

\footnotetext{
${ }^{1}$ The need-based framework also accounts for the possibility that attractiveness of locations has an impact on a need's size through induction. However, we ignore this aspect for the present measurement purpose.
} 
Again following the needs-based theory, the size of each need after having completed the $j$ th (activity or travel) episode is given by:

$$
B_{i}^{t_{j}}=\left\{\begin{array}{ll}
B_{i}^{t_{j-1}}+\Delta B_{i j} & \text { if } \Delta B_{i j} \neq 0 \\
f\left(B_{i}^{t_{j-1}}, T_{j}, \beta_{i}, B_{i}^{\max }\right) & \text { otherwise }
\end{array}\right\}
$$

where $t_{j}$ is the end time of episode $j, T_{j}$ is the duration of episode $j, f$ is a need-growth function, $\beta_{i}$ and $B_{i}{ }^{\max }$ are parameters of the need-growth function, and, as before, $\Delta B_{i j}$ is the change of need $i$ caused by activity $j$. As the two conditions in this equation reflect, a need is either affected by activity $j$ or not. If it is not affected, the need increases as a function of the length of the episode $\left(T_{j}\right)$ and a growth rate that is dependent on the existing size of the need before the activity, a maximum size, $B_{i}{ }^{\text {max }}$, and a growth speed, $\beta_{i}$. As for the form of $f$, we assume a logistic growth function where growth accelerates in the low range and de-accelerates in a high range of the existing need such that need size is bounded by a minimum (of zero) and some maximum $\left(B_{i}^{\max }\right)$. The maximum and the growth rate at the inflection point $\left(\beta_{i}\right)$ are parameters of the function that differ between needs and may differ between individuals or households as well.

Finally, the impact of an activity is modeled as follows:

$$
\Delta B_{i j}=\left\{\begin{array}{ll}
b_{i j} B_{i}^{t_{j-1}} & \text { if } b_{i j}<0 \\
b_{i j}\left(B_{i}^{\max }-B_{i}^{t_{j-1}}\right) & \text { if } b_{i j}>0 \\
0 & \text { if } b_{i j}=0
\end{array} .\right.
$$

where $b_{i j}$ is the so-called potential of activity $j$ to satisfy need $i$. As implied by this equation, an activity either has a decreasing $(b<0)$, an increasing $(b>0)$ or no impact $(b=0)$ on the need considered. If it has an impact, the potential indicates the change of the need as a proportion of the existing need $(b<0)$ or as a proportion of the maximally possible increase given the existing size and maximum size of the need $(b>0)$. This potential is a function of properties of the activity as follows:

$$
b_{i j}=g\left(T_{j}, \alpha_{j}^{D}, \beta_{j}^{D}\right) h\left(X_{j}, \alpha_{j}^{A}, \beta_{j}^{A}, \delta_{i j}\right) b_{i j}^{0}
$$

where $-1 \leq b_{i j}{ }^{0} \leq 1$ is a so-called base potential, $g$ is a duration function and $h$ is an attribute function. The Greek symbols represent parameters of functions (explained below). Both the duration and attribute function return a value between zero and one and, thus, indicate the proportion of the base potential realized by the activity. The base potential is a fixed property of the activity which defines the maximum impact it can have and the sign of the impact.

A particular important issue for the measurement purpose is the question how the duration and attribute functions are specified. Following again the basic theory, we assume a logistic function for the impact of duration as follows:

$$
g\left(T_{j}, \alpha_{j}^{D}, \beta_{j}^{D}\right)=\frac{1}{1+\exp \left(\beta_{j}^{D}\left[\alpha_{j}^{D}-T_{j}\right]\right)}
$$

This describes an increasing function of duration with an asymptotic minimum of zero and an asymptotic maximum of one. Alpha and beta parameters are activity and 
possibly individual-specific. Alpha represents the duration where the marginal potential is at a maximum and beta represents the slope of the function at that point. Thus, activities that take long time before becoming effective have a large value of alpha (and vice versa) and activities that have relatively fixed durations have a large value of beta. For the measurement purpose, we assume the following attribute function:

$$
h\left(X_{j}, \alpha_{j}^{A}, \beta_{j}^{A}, \delta_{i j}\right)=\frac{1}{1+\exp \left(\beta_{j}^{A}\left[\alpha_{j}^{A}-V\left(X_{j}, \delta_{i j}\right)\right]\right)}
$$

where $V$ is an attribute utility function The exponential form makes sure that the function is bounded to a zero-one range. The attribute utility function is defined in a straight-forward way as:

$$
V\left(X_{j}, \delta_{i j}\right)=\delta_{i j} X_{j}^{\prime}
$$

where delta is an attribute vector of weights and $X$ is an attribute vector of values of the location/route where the activity/trip is conducted.

\section{Measuring the Quality of Urban Environments}

The above set of equations allows us to calculate the utility of an activity pattern by calculating need changes caused by each episode sequentially in the order in which they occur in the pattern, as schematically illustrated in Fig. 1. The model has several noteworthy properties. First, the utility an individual derives from a given activity pattern depends on the existing needs of the individual at the start of the day (or, more generally, at the start of the period monitored). Needs may vary across individuals possibly related to factors we can actually measure in a survey such as socio-economic variables (e.g., life cycle), household composition (e.g., being single or not) and characteristics of the dwelling (e.g., having a garden or not). For example, individuals having a garden or living in a green neighborhood may have smaller green needs when they leave from home and derive less utility from green alongside routes and at activity locations than others. Thus, the model is sensitive (in

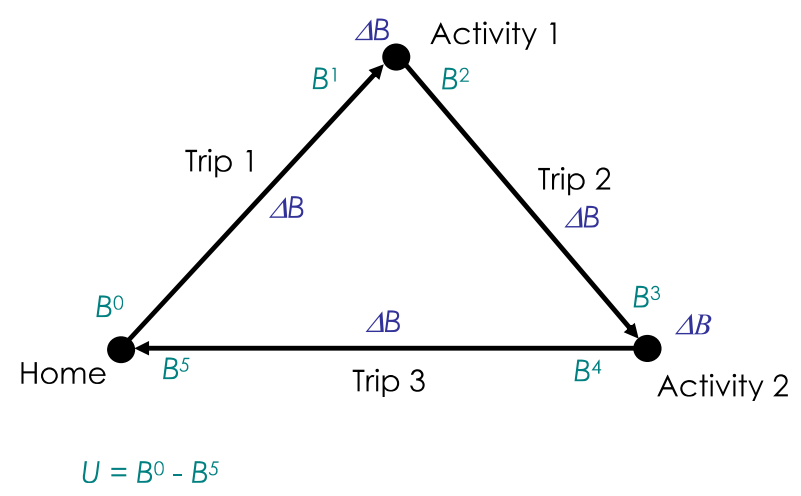

Fig. 1 Graphical illustration of measuring need-satisfaction for a tour including two activities 
principle) to socio-economic composition as well as situational factors of a studied population.

Second, the model is sensitive to the interaction between activities and attributes of locations and routes. Keeping everything else constant, the longer the exposure to attributes, in a given mode of behavior (activity or transport mode), the larger the overall impacts of these attributes on needs and, hence, on perceived utility, while marginal impacts typically decrease due to saturation. This holds not just within activities but also between activities. For example, traveling a green route to work may decrease the utility derived from green facilities at facility locations. As another example, the utility an individual associates with a covered passageway for shopping may be dependent on whether a bike or car is used for the trip to the location. Such interaction effects are not limited to activities within a same tour, but can also occur between tours ${ }^{2}$, between days (if a multi-day period is considered) or between persons (if the wider household context is considered).

\section{Representation of Activity Patterns in a GIS}

It follows from the foregoing that utilities individuals derive from their activity patterns are a complex function of the organization of activities in space and time. GIS are ideally suited to spatially represent individual activity patterns and calculate attributes of routes and locations involved in these patterns by means of buffer analysis and overlay analysis combining several layers of attribute data. In this section, we assume the activity patterns of a sample of individuals in a study area as given and consider the question how these activity patterns can be represented using existing GIS-tools. The proposed method is implemented in the GIS-software TransCAD (www.caliper.com) which offers useful tools especially in the area of transport systems and network analyses.

The definition and measurement of attributes of locations and routes are important issues for capturing the above notions. We represent an activity pattern as a path through a transportation network, usually a road network. An activity is described as staying at a node on the network, which corresponds to the activity location. A trip is described as a sequence of links traversed during traveling. A tour is a sequence of trips, including one or more stops, which starts from a base location and ends at the same base location. In the following we will assume that home is always the base location. We use the terms route and path to denote the sequence of links traversed on a trip and the sequence of links traversed on a tour, respectively. Thus, in this terminology, a path consists of a sequence of routes and a route consists of a sequence of links.

Given the measurement purpose, we define attributes of locations (e.g., nodes) and routes generally by means of aggregation of attributes of objects that fall inside a buffer of pre-defined size around the node or link, where objects are either polygons, points or lines. The buffer size should reflect the distance over which an attribute exerts its influence either through being visible or by the action radius of the activity.

\footnotetext{
$\overline{{ }^{2}}$ A tour is a chain of trips starting at a base location — e.g., home — and ending at a base location including one or more activity locations.
} 
This may be dependent on activity type or transport mode. For example, a shopping activity may have a wider action radius than an indoor activity and field of vision may be larger for a slow mode than car. Aggregation may simply be based on calculating a sum or average of the quantity represented by the attribute (e.g., number of objects of a certain type or total area of an object of a certain type). To describe routes, a further aggregation is needed, namely an aggregation of attributes across the links included.

\section{A Case Study}

In this section, we discuss an application to illustrate the method and explore its sensitiveness to spatial planning scenarios. As a case, we consider a particular dimension of needs, namely the (time-varying) need for being in a green environment. Although the intensity may vary between individuals, we assume that this need is universal. Activity patterns extracted from activity diaries collected in the Eindhoven region (the Netherlands) in 2001 are assumed as given and we measure for each pattern the extent to which a green need is satisfied during execution of the pattern given the spatial distribution of green in the Eindhoven region. To show the sensitivity of the method, we compare measurement results between the existing situation and a hypothetical scenario of changes in the spatial distribution of green area in the city.

\section{Activity Pattern Data}

An existing activity diary data set was used to reveal activity patterns of a sample of individuals. The data were collected in the Eindhoven region in 2001. All persons of 12 years or older in households were asked to keep a diary of their activities and related travel for 2 consecutive days. Days were assigned randomly to households such that at sample level all days of the week are equally represented. After cleaning for the present measurement purpose, the total dataset includes 619 person-day diaries. In total 1,007 tours were reported in these diaries, which comes down to an average of 1.63 tours per person-day. On average a tour includes 2.56 trips and 1.63 activities implying that a proportion of trips include multiple activities. For a more detailed description of the dataset and survey method, readers are referred to Kemperman et al. (2006).

In the interest of computation time, a random subset of 94 person-days including 160 tours was selected for the present analysis, which should be representative for the entire sample. The selected tours cover 345 paths. Figure 2 shows the tours (route systems) on a map of the area. As the figure indicates, the tours cover the area of the Eindhoven region to a sufficient extent. Activities were classified with respect to their supposed interaction with green attributes of the environment into outdoorgreen-recreation activities, outdoor-other activities and indoor activities (out-ofhome or in-home). Outdoor activities include activities such as picking-up or dropping-off persons, shopping, visiting a post-office, etc., whereas indoor activities include working, school, paying someone a visit, indoor sports, etc. Transport modes were classified based on the same consideration into fast modes (car, bus, train, etc.) and slow modes (bike, walking, etc.). 


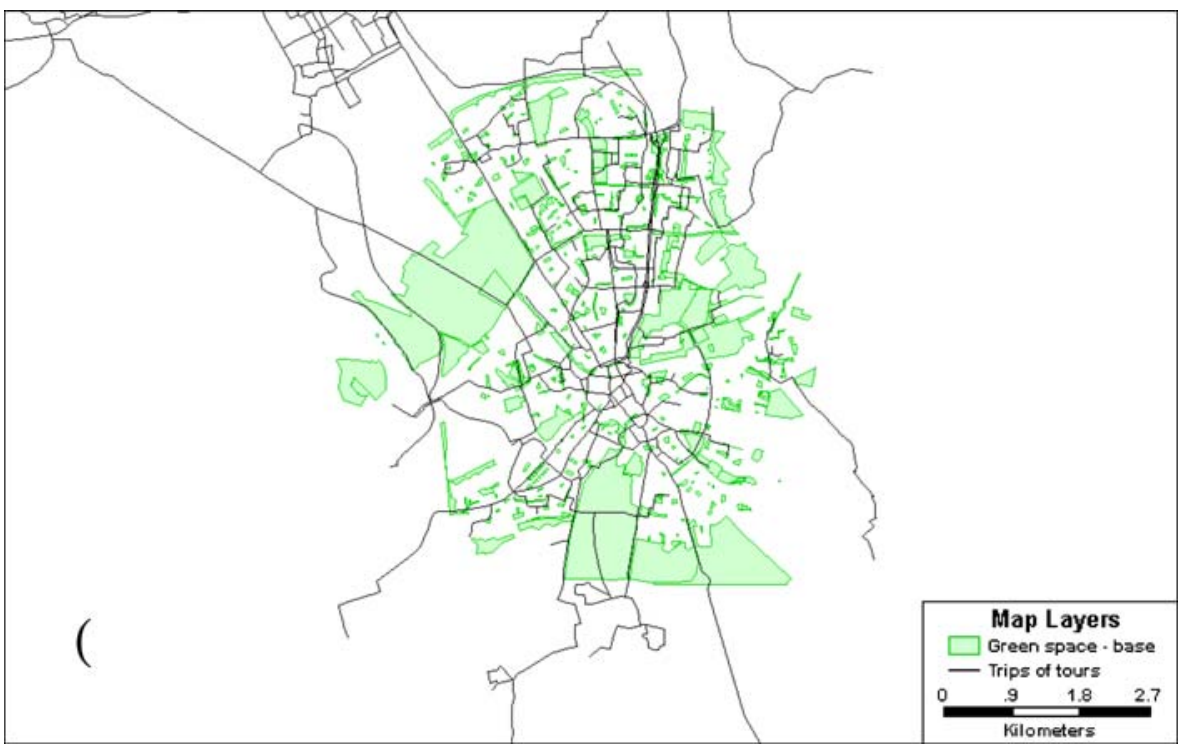

Fig. 2 Map of route systems (activity patterns) and green areas (parks) in the Eindhoven region

Study Area and Spatial Analysis

As attributes of links and locations, the total area of green land of different types within buffers around links and locations were calculated. Considering the illustration purpose, buffer widths were not differentiated by activity type or transport mode. A buffer width of $100 \mathrm{~m}$ was assumed for links and a buffer width of $500 \mathrm{~m}$ was used for locations reflecting the assumption that interaction with the environment stretches out to a wider area when being at a location than when traveling on a link. Two different types of green land were distinguished, namely neighborhood parks and higher-order parks. Generally, higher-order parks cover a larger area and offer more diversity of green vegetation. Figure 2 shows the spatial distribution of green areas (neighborhood and higher-order) in the city. In total there are 374 neighborhoods and 53 higher-order parks in the study area. The activity data includes 179 unique activity locations, 100 of which include neighborhood green in their buffer (of on average 4.5 ha wide) and 82 of which include higher-order green in their buffer (of on average 7.8 ha wide). On the other hand, the activity data includes 345 unique paths. Two hundred nine paths include neighborhood green (on average $3.3 \mathrm{ha}$ ) in buffers and 166 paths have higher-order green in buffers (on average $7.4 \mathrm{ha}$ ).

The scenario we consider assumes that part of the neighborhood parks in the urban area of Eindhoven disappear as a consequence of developing these areas for housing to accommodate a hypothetical increase in demand for housing in the city. Higher-order parks are not affected by the scenario. As a consequence of the scenario, activity locations including neighborhood green (in buffers) decreases from 100 to 87 locations and the average size of neighborhood green area of these locations drops from 4.5 ha to 1.3 ha. As for paths, the number of routes with 
neighborhood green decreases from 209 to 182 paths and the average size of neighborhood green area of these paths drops from 3.3 ha to 1.2 ha.

\section{Measurement Results}

Equations 1-7 were applied to calculate a utility for each activity-pattern on a person-day basis. Arbitrarily, the initial size of a need for green at the start of the day of each individual was set to 100 units. Theoretically, a need for green could grow during the day or be induced by certain activities. However, it was assumed that activities implemented by the individual in the environment can only have a satisfying or no impact on the need and that when an activity has no impact the need stays constant (no autonomous growth). We emphasize that the initially assumed size of the need can be set fully arbitrarily as it just serves to set a scale (for the need dimension and, hence, utility). Parameters of the duration function were set as: $\alpha^{\mathrm{D}}=$ 10 and $\beta^{\mathrm{D}}=0.45$ and parameters of the attribute function were set as: $\alpha^{\mathrm{A}}=5$ and $\beta^{\mathrm{A}}=\beta^{\mathrm{D}}=0.45$. Furthermore, base potentials of activities were set as $b^{0}=0$ for indoor activities, $b^{0}=-0.5$ for outdoor activities where green recreation is not a primary purpose and $b^{0}=-1$ for outdoor activities where green recreation is a primary purpose. The base potentials for transport modes were set as $b^{0}=-0.8$ for slow modes and $b^{0}=-0.5$ for fast modes. High-order green was weighted more heavily than neighborhood green according to the ratio $6: 4$ (i.e.: $\delta_{\mathrm{h}-\mathrm{o}}=0.6, \delta_{\mathrm{nbh}}=0.4$ ).

Table 1 shows the analysis of an arbitrary activity pattern, as an example. In this activity pattern, the individual made two tours on the observed day. The first tour was conducted by a slow mode and included a single indoor activity. The second tour was conducted by a fast mode and combined two activities - an indoor and outdoor (non-green) activity. The activities were conducted at the same location (node of the network) implying that measured travel time equals zero. The last two columns in the table show the size of the green need before and after each episode.

Table 1 Measurement results for an activity-pattern example

\begin{tabular}{|c|c|c|c|c|c|c|c|}
\hline \multirow[t]{2}{*}{ Tour nr } & \multirow[t]{2}{*}{ Episode } & \multirow[t]{2}{*}{ Mode/Type } & \multirow{2}{*}{$\begin{array}{l}\text { Duration } \\
\text { (min) }\end{array}$} & \multicolumn{2}{|c|}{ Size of green (ha) } & \multicolumn{2}{|c|}{ Need size } \\
\hline & & & & Neighbhd & High-order & Start & End \\
\hline \multirow[t]{4}{*}{ Tour 1} & Travel & Slow mode & 15 & 4.37 & 3.8 & 100.0 & 71.6 \\
\hline & Activity & In-door & 15 & 5.61 & 0 & 71.6 & 71.6 \\
\hline & Travel & Slow mode & 30 & 4.37 & 3.8 & 71.6 & 49.1 \\
\hline & Home & In-door & & 4.65 & 8.27 & 49.1 & 49.1 \\
\hline \multirow[t]{6}{*}{ Tour 2} & Travel & Fast mode & 30 & 2.9 & 11.27 & 49.1 & 29.8 \\
\hline & Activity & In-door & 120 & 0.71 & 0 & 29.8 & 29.8 \\
\hline & Travel & Fast mode & 0 & 0 & 0 & 29.8 & 29.8 \\
\hline & Activity & Out-door & 30 & 2.66 & 0 & 29.8 & 27.6 \\
\hline & Travel & Fast mode & 30 & 2.54 & 11.27 & 27.6 & 16.9 \\
\hline & Home & In-door & & 4.65 & 8.27 & 16.9 & 16.9 \\
\hline
\end{tabular}


Across all episodes of the two tours the green need has dropped from 100 to 16.9 units meaning that the utility of the activity pattern equals $83.1(=100-16.9)$ units. As a consequence of assumed settings of base potentials, indoor activities do not contribute to this utility at all and the outdoor activity only to a modest extent (given its duration and size of green in the proximity of the location). The trips by slow mode contribute most strongly to the utility as a result of its base potential and size of green area along side the route traveled (in a $100 \mathrm{~m}$ band from each side). The example illustrates that the utility derived is the result of interactions between characteristics of the activity pattern (activity type, durations, places), on the one hand, and characteristics of the environment, on the other (green alongside routes and in proximity of locations).

The average utility across all activity patterns in the existing situation equals 40.2 units. The relatively high standard deviation of 29.4 units indicates that the model is rather sensitive to differences between activity patterns. After implementing the scenario, the average utility decreases to 35.2 units (with a standard deviation of 28.0 units). Thus, on average, individuals when they implement their activities in the city would experience a utility loss of 5.0 units or $12.4 \%$ of the existing level. We can also put this by saying that, as a consequence of the scenario, the remaining need for green at the end of an average day of an average individual increases from $59.8 \%$ to $64.8 \%$, as predicted by the model. Although settings of parameters of the model are set heuristically, this analysis shows that the model is sensitive to attributes of the environment.

The chosen parameter settings are somewhat arbitrary. To assess the sensitivity of the model for parameter settings, Table 2 shows results of the same utilitymeasurements before and after implementing the scenario for different settings where one parameter is varied at a time. The first row represents the result, as discussed above, of the base setting. The second two rows represent the results where the alpha parameter of the duration function is changed first to $\alpha^{\mathrm{D}}=5$ and next to $\alpha^{\mathrm{D}}=15$. The next two rows show the results where this parameter is reset to the base level, i.e. $\alpha^{\mathrm{D}}=10$ and the beta parameter of the duration function is set first to $\beta^{\mathrm{D}}=0.3$ and next to $\beta^{\mathrm{D}}=0.6$. In subsequent rows the alpha and beta parameters of the

Table 2 Results of sensitivity analysis

\begin{tabular}{lccc}
\hline Setting & Utility before mean (stdev) & Utility after mean (stdev) & \% utility loss \\
\hline Base & $40.2(29.4)$ & $35.2(28.0)$ & 12.4 \\
$\alpha^{\mathrm{D}}=5$ & $45.3(29.2)$ & $40.0(28.5)$ & 11.5 \\
$\alpha^{\mathrm{D}}=15$ & $33.3(28.8)$ & $28.8(26.8)$ & 13.6 \\
$\beta^{\mathrm{D}}=0.3$ & $39.9(29.1)$ & $35.0(27.8)$ & 12.3 \\
$\beta^{\mathrm{D}}=0.6$ & $40.2(29.6)$ & $35.2(28.2)$ & 12.5 \\
$\alpha^{\mathrm{A}}=2.5$ & $55.7(28.6)$ & $51.7(28.1)$ & 7.1 \\
$\alpha^{\mathrm{A}}=7.5$ & $26.7(26.8)$ & $22.4(25.2)$ & 16.3 \\
$\beta^{\mathrm{A}}=0.3$ & $45.6(26.6)$ & $42.0(25.7)$ & 7.9 \\
$\beta^{\mathrm{A}}=0.6$ & $36.5(31.9)$ & $30.6(30.3)$ & 16.2 \\
\hline
\end{tabular}


attribute functions are varied similarly as $\alpha^{\mathrm{A}}=2.5$ and $\alpha^{\mathrm{A}}=7.5$, and as $\beta^{\mathrm{A}}=0.3$ and $\beta^{\mathrm{A}}=0.6$, respectively. As the results indicate, the model is quite robust in terms of the assessment of the relative decrease in utility ( $\%$ loss) individuals on average would experience in the scenario. The utility loss varies between $7.1 \%$ and $16.3 \%$ across the parameter settings. This means that in a rather wide range of settings the model displays considerable sensitivity to the spatial changes. The results also indicate that variation in settings of the attribute function has a higher impact than variation in settings of the duration function, as could be expected. Across the variation in duration parameters, the loss varies in a much more limited range from $11.5 \%$ to $12.4 \%$.

\section{Conclusions and Discussion}

In this paper, we proposed a new approach to measuring quality aspects of an urban environment regarding the implementation of the daily activities of individuals. The approach is based on a needs-based theory of activity generation, which states that daily activities of individuals/households are driven by a set of universal needs. The needs-based model defines the mutual relationships between activities, needs and utilities (Arentze and Timmermans 2006, 2009). Attributes of locations where activities are conducted and routes used to travel to the locations influences the ability of activities to satisfy particular needs. We showed how the model can be used using standard GIS tools and a micro-simulation approach to measure the extent to which an environment supports activities. As an illustration, the case study focused on green attributes in relation to a peoples need for being in a (natural) green environment every now and then. Other need-dimensions, such as for example to have physical exercise, to socially interact with other people, to entertain oneself, to have control over one's actions and overview of the environment, have in addition been established in empirical psychological studies. All existing need dimensions, as far as they are affected by attributes of a space-time environment, can be taken into account simultaneously in the measurement approach proposed in this study.

The measurement method is complementary to existing accessibility-oriented approaches. The latter approaches focus on the amount of effort needed to reach facility locations for activities given a spatial distribution of facilities in the environment. The most basic methods measure accessibility as the effort (e.g., generalized travel costs) needed to reach a nearest facility for a particular activity or as the number of facility locations that can be reached within a given amount of effort. More elaborated methods also take subjective preferences for location attributes (e.g., consumer surplus) and/or limited time budgets of individuals (e.g., space-time prisms) into account, but depart from the same conceptualization. The method proposed in the present study focuses on a complementary quality aspect of urban environments. We conceptualized activities of individuals as a path through space and time. In traversing this path, an individual is exposed to attributes of locations and routes. The interaction between activities, attributes, and the duration of exposure determines the extent to which specific needs are fulfilled and utilities experienced. This conceptualization is more akin to exposure models of measuring physical aspects of an environment. Thus, complementary to effort, the new method 
highlights attributes of the environment that affect other dimensions of an individual's well being. When this additional dimension of urban environments is taken into account, traveling, for example, is no longer viewed exclusively as an effort to overcome a spatial separation between activity locations. As the case study illustrated, travel in this framework is also regarded as an activity that may satisfy particular needs (in the illustration a need for green recreation). The positive utility of travel has also been emphasized by other authors (Mokhtarian 2005).

The combination of activity-based accessibility measurements (in the constraintsbased approach) and the newly proposed needs-based measurement, thus, provides a more complete view on what a specific urban environment has to offer individuals given their activity agendas. Constraints-based models assume an (daily) activity program as given and use some combinatorial algorithm to generate an exhaustive set of feasible implementations of the activity program, given a space-time setting. The number of feasible patterns can be used as a performance indicator of that setting. When a generalized cost function of traveling is available, the minimum effort across feasible activity programs could be taken as an alternative measure of space-time opportunities. Regardless of the details of the approach, the constraintsbased approach relies on a micro-simulation of activity patterns in space and time. In this study, we showed for a given simulated pattern how a needs-based utility can be conceptualized and quantified as a function of attributes of the environment. Combining the latter measure with an accessibility measure, thus, enables the analyst to take additional quality aspects of an environment into account. In a case study, where we focused on a need for green, we showed that the measure is sensitive for the kind of changes that may be involved in spatial policies in a wide range of possible parameter settings.

Several problems remain for future research. First, the application described in this study focused on a need for green. To capture a wider range of need dimensions, empirical research is needed first to identify all needs that are relevant in the context of daily activities (and travel) and second to identify and measure attributes of an urban environment that impacts on each need. The CNET cognitive mapping technique developed in Dellaert et al. (2008) and Arentze et al. (2008) seem to be ideally suited to reveal attribute and need dimensions through a questionnaire. Second, having identified the need dimensions, estimation of the model's parameters can be undertaken. Parameters that need to be estimated concern 1) the relative importance of each need (i.e., the maximum value of the need), possibly, differentiated based on socio-economic attributes and characteristics of the residential environment and 2) activity specific parameters of environmental attributes in need-satisfaction functions.

The task of estimating such parameters can draw on extensive literature and long history of estimating utility functions based on choice observations in the field of discrete choice modeling. Furthermore, in more recent studies estimation methods have been developed and explored specifically in the needs-based framework. Arentze et al. (2009) developed and tested an approach to estimate parameters of a similar needs-based model using loglikelihood estimation and activity diary data of the same kind as used in this study. Their results suggest that except for a single utility-scale parameter all need-function parameters can be identified. Ettema et al. (2009) developed and validated a method to estimate parameters of a similar needs- 
based model based on activity duration and activity frequency data. Thus, these studies reveal promising ways to estimate the duration, attribute and need functions based on data that are readily available or can be collected against low costs. The present study showed that the model is sensitive to environmental variables for a wide range of possible settings of parameters. With further advancement of estimation methods, the parameters that currently were set heuristically can be fine-tuned to values that reflect actual preferences and values of individuals.

Open Access This article is distributed under the terms of the Creative Commons Attribution Noncommercial License which permits any noncommercial use, distribution, and reproduction in any medium, provided the original author(s) and source are credited.

\section{References}

Arentze, T. A., \& Timmermans, H. J. P. (2006). A new theory of dynamic activity generation. In Proceedings of the $85^{\text {th }}$ Annual Meeting of the Transportation Research Board, Washington, D.C., (CD-ROM: 20 pp).

Arentze, T. A., \& Timmermans, H. J. P. (2009). A need-based model of multi-day, multi-person activity generation. Transportation Research B, 43, 251-265.

Arentze, T. A., Borgers, A. W. J., \& Timmermans, H. J. P. (1994a). Geographical information systems and the measurement of accessibility in the context of multipurpose travel: a new approach. Geographical Systems, 1, 87-102.

Arentze, T. A., Borgers, A. W. J., \& Timmermans, H. J. P. (1994b). Multistop-based measurements of accessibility in a gis environment. International Journal of Geographical Information Systems, 8 , 343-356.

Arentze, T. A., Dellaert, B. C. G., \& Timmermans, H. J. P. (2008). Modeling and measuring individuals' mental representations of complex spatio-temporal decision problems. Environment and Behavior, 40 (6), 843-869.

Arentze, T. A., Ettema, D, \& Timmermans, H. J. P. (2009). Estimating a model of dynamic activity generation based on one-day observations: Method and results. In Proceedings of the 88th Annual Meeting of the Transportation Research Board, Washington, D.C., (CD-ROM: 18 pages).

Ben-Akiva, M., \& Lerman, S. R. (1978). Disaggregate travel and mobility-choice models and measures of accessibility. In D. Hensher \& P. R. Stopher (Eds.), Behavioural travel modelling, pp. 654-679. London: Helm.

Breheney, M. J. (1978). The measurement of spatial opportunity in strategic planning. Regional Studies, $12,463-479$.

Coelho, J. D., \& Wilson, A. G. (1976). The optimum location and size of shopping centres. Regional Studies, 10, 413-421.

Dijst, M., de Jong, T., \& van Eck, J. R. (2002). Opportunities for transport mode change: an exploration of a disaggregated appraoch. Environment and Planning B, 29, 413-430.

Dalvi, M. Q. (1978). Behavioural modelling, accessibility, mobility and need: Concepts and measurement. In D. Hensher \& P. R. Stopher (Eds.), Behavioural travel modelling, pp. 639-653. London: Croom Helm.

Dellaert, B. C. G., Arentze, T. A., \& Timmermans, H. J. P. (2008). Shopping context and consumers' mental representation of complex shopping trip decision problems. Journal of Retailing, 84, 219-232.

Ettema, D., Arentze, T. A., Timmermans, H. J. P. (2009). Need-based activity generation and duration model: Empirical tests. In Proceedings of the $88^{\text {th }}$ Annual Meeting of the Transportation Research Board, Washington, D.C., (CD-ROM: 27 pp).

Ingram, D. R. (1971). The concept of accessibility: a search for an operational form. Regional Studies, 5, 101-107.

Jones, P. M., Dix, M. C., Clarke, M. I., \& Higgie, I. G. (1983). Understanding travel behaviour. Hants: Aldershot.

Kemperman, A. D. A. M., Arentze, T. A., \& Timmermans, H. J. P. (2006). Social commitments and activity-travel scheduling decisions. Transportation Research Record, 1977, 242-249. 
Kim, H. M., \& Kwan, M. P. (2003). Space-time accessibility measures: a geocomputational algorithm with a focus on the feasible opportunity set and possible activity duration. Journal of Geographical Systems, 5, 71-91.

Koenig, J. G. (1980). Indicators of urban accessibility: Theory and application. Transportation, 9, 145172.

Kwan, M.-P. (1998). Space-time and integral accessibility measures: a comparative analysis using a pointbased framework. Geographical Analysis, 30, 191-216.

Kwan, M.-P. (1999). Gender, the home-work link, and space-time patterns of nonemployment activities. Economic Geography, 75, 370-394.

Lenntorp, B. (1978). A time-geographic simulation model of individual activity programmes. In T. Carlstein, D. Parkes \& N. Thrift (Eds.), Human activity and time geography (vol. 2, pp. 162-180). London: Arnold.

Leonardi, G. (1978). Optimum facility location by accessibility maximizing. Environment and Planning A, 10, 1287-1305.

Miller, H. J. (1999). Measuring space-time accessibility benefits within transportation networks: Basic theory and computational procedures. Geographical Analysis, 31, 187-212.

Mokhtarian, P. L. (2005). Travel as a desired end, not just a means. Transportation Research A, 39, 63-69.

Wachs, M., \& Kumagai, T. G. (1973). Physical accessibility as a social indicator. Socio-Economic Planning Science, 7, 437-456.

Weber, J. (2003). Individual accessibility and distance from major employment centers: an examination using space-time measures. Journal of Geographical Systems, 5, 51-70. 